\title{
Numerical noise in satellite laser ranging data processing
}

\author{
J. Blazej \& I. Prochazka \\ Czech Technical University in Prague, Czech Republic
}

\begin{abstract}
Satellite laser ranging is a highly accurate measuring technique providing the accurate range to the retroreflector equipped Earth satellites. It operates on a classical radar principle. Measuring the time interval between the pulse transmittal and reception, and considering the speed of light and the way of its propagation, the target distance may be evaluated. The picosecond laser pulses together with highly precise timing systems and optical detectors enable resolution and accuracy of the entire ranging system to an accuracy of several millimetres. To demonstrate the ultimate precision of satellite laser ranging two different and independent algorithms were used for ranging data processing. The internal consistency and the numerical noise of the data reduction, fitting and normal point forming procedure have been tested. The completed experiment demonstrates the ultimate normal point precision at the level of 2.5 picoseconds, that corresponds to $0.37 \mathrm{~mm}$ in the range of the retroreflector equipped satellite in space. The key contributor to this value is the interpolation used in the satellite orbit modelling procedure.
\end{abstract}

Keywords: picosecond ranging, orbit modelling.

\section{Introduction}

The testing of precision limits of algorithms used is interesting for any technique whose precision during recent years is improving by technology reasons without changing of the data processing algorithm. One of these is the Satellite Laser Ranging (SLR). The SLR is a highly accurate measuring technique providing accurate range to retroreflector equipped Earth satellites. It operates on a classical radar principle. Measuring the time interval between the pulse transmission and reception and considering the speed of light and the way of its 
propagation, the target distance may be evaluated. The picosecond laser pulses together with highly precise timing systems and optical detectors enable resolution and accuracy of the entire ranging system to a range of several millimetres. The results of SLR are used, among others, for the determination of terrestrial reference frame and the product of the universal gravitational constant and the Earth mass, which represent one of the fundamental constants in physics, for details see Pearlman et al [1]. In this view, the SLR serves as a fundamental technique to calibrate other measurements. That is why the precision and accuracy of the SLR data itself is a critical issue.

\section{Data processing}

The SLR is a technique developed over more than thirty years. One unpropitious consequence of this fact is the squeezing by standard data interchanging formats of a data processing algorithm. To reduce the requirements on data transfer and archiving, to simplify the data analysis procedures, and to simplify the data use, the individual (so-called single shot) SLR measurements are compressed into socalled normal points. The data time series are divided into time slots - time bins of common predefined size of $5 \div 300$ seconds, depending on the satellite orbit altitude. The individual ranging data within one bin are fitted and averaged and at the end are represented by a single range called the normal point.

The goal of this paper is to compare two independently implemented algorithms to help identify error source limitations of the normal point precision.

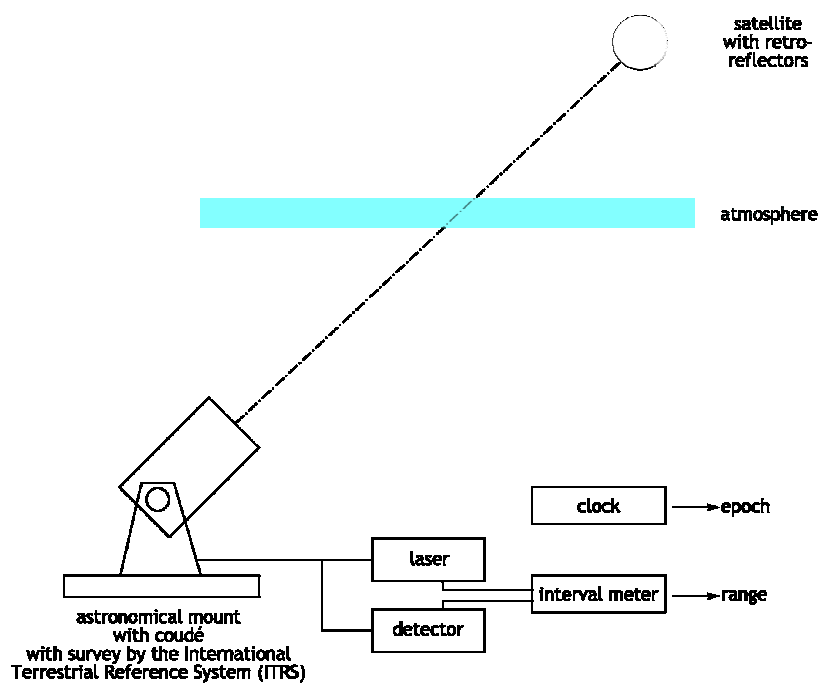

Figure 1: The schematic diagram of SLR principle, time interval between laser pulse transmission and detection is measured together with time of measurement - epoch. 


\section{Experiment}

The main factors limiting the normal point precision are: the overall stability of the laser ranging chain and the precision of the data reduction and fitting procedure. The laser ranging hardware and its stability were characterized by means of the Portable Calibration Standard in numerous trials, see Hamal et al [2]. The best laser ranging stations achieve long term system bias stability of the order of picoseconds in time that corresponds to the $0.1 \mathrm{~mm}$ in range. The internal consistency and the numerical noise of the data reduction, fitting and normal point forming procedure were tested by the following experiment.

The SLR raw ranging data were processed, fitted, and normal points were formed using two different and independent algorithms.

\subsection{Graz SLR data reduction}

The orbit is modelled by a IRVINT Sinclair [3] integrator providing 1 minute $\mathrm{x}, \mathrm{y}, \mathrm{z}$ coordinates, 8-points Lagrange interpolation and topocentric conversion, and optional manual range and time bias tuning. The generated orbit is subtracted from range data and the residuum series is fitted by a polynomial fitting (standard scheme, degree 5-10, optionally 20) data screening and manual editing (validation). All interchange data files are in MERIT2 data format, i.e. with 1 picosecond granularity.

\subsection{Portable Calibration Standard data reduction}

This software package, see Hamal et al [2], consists of orbit integration using the Herstmonceux RGO scheme providing 1 minute $\mathrm{x}, \mathrm{y}, \mathrm{z}$ coordinates, 8-points Lagrange interpolation and topocentric conversion, automated range bias, time bias, and device universal time tuning procedure. The generated orbit is subtracted from the range data and residua are iterative polynomial fitting with fully automated data editing. In this data reduction package, the processing consists of a sequence of individual programs, the data are passed from one program to another via a formatted data file with the least significant digit of 1 picosecond, and thus the rounding is implemented 3 to 5 times consequently.

\subsection{Results}

The ranging data, described in Prochazka and Kirchner [4], from SLR station in Graz, Austria, were used. Segments of the ERS-2 satellite ranging with the data rate of about 750 echoes per second, acquired from October 2003 to January 2004 were selected. The echoes from the closest corner retroreflector were selected to eliminate the influence of the target completely from the process. The single shot precision achieved in the tested data series was 18 to 20 picoseconds rms, that corresponds to a 2.5 to $3.0 \mathrm{~mm}$ range precision of a single shot measurement. 
The range residuals of individual ranges (single shot) were compared for all measurements. The difference between the computed ranging residuals on a shot by shot basis indicates the estimate of the data processing procedure. The computed differences have the characteristics displayed in figure 2. One can see a slowly varying component with a varying period of about 15 seconds and an amplitude of 2.0 picoseconds, and random spread within 1.5 picoseconds. The slowly varying component was attributed to the interpolation, the random component to the numerical noise of the computation and to the rounding process. The slowly varying component including absolute shift of the order of 2 picoseconds is the dominant contributor.



Figure 2: Data residuals comparison of two described algorithms. The difference is computed for each shot, the integral value for 0.2 second is plotted.

From range residuals, results were calculated. The numerical experiments of Prochazka and Kirchner [4] showed the deviation from the ideal model in the case when about 75 individual range measurements are compressed into one normal point with the corresponding normal point precision of $2.5 \mathrm{ps}$. The second break point occurred when compressing more than 2000 points with the corresponding normal point precision of $1 \mathrm{ps}$. These two break points correspond perfectly to the two limiting factors identified as the interpolation and rounding errors. 


\section{Conclusion}

The completed experiment demonstrates the ultimate normal point precision at the level of 2.5 picoseconds, that corresponds to $0.37 \mathrm{~mm}$ in the range of the retroreflector equipped satellite in space. This limit value is negligible in comparison to the signatures of actually used satellites. The key contributor to this value is the interpolation used in the orbit modelling procedure. The last significant digit in order of one picosecond is acceptable in today's SLR data processing formats.

\section{Acknowledgements}

The authors are grateful to Georg Kirchner and Franz Koidl, Graz SLR station. This research was supported by the research framework MSM6840770015 of the Ministry of Education of the Czech Republic.

\section{Reference}

[1] Pearlman, M.R., Degnan, J.J., and Bosworth, J.M., "The International Laser Ranging Service", Advances in Space Research, Vol. 30, No. 2, pp. 135-143, July 2002.

[2] Hamal, K., Prochazka, I., Blazej, J., Kirchner, G., Schreiber, U., Riepl, S., Sperber, P., Gurtner, W., Appleby, G., Gibbs, P., Yang Fumin, Neubert, R., and Grunwaldt, L., Satelite Laser Ranging Portable Calibration Standard Missions 1997-2002, In Abstract Book 'Geophysical Research Abstracts' Volume 5, 2003, EGS - AGU - EUG Joint Assembly 2003, Nice, France, p. 243.

[3] Sinclair, A, TIRV Reference System, [online], [cit. 02/03/07], http://ilrs.gsfc.nasa.gov/products_formats_procedures/predictions/tirv.htm 1.

[4] Prochazka, I., Kirchner, G., Numerical noise in satellite laser ranging data processing, Boletin ROA No. 4/2004, ed. J.M.Davila, San Fernando: Real Instituto Observatorion de la Armada, ISSN 1131-5040, 2004. 